\title{
Improving screening for malnourished children at high risk of death: a study of children aged 6-59 months in rural Senegal
}

\author{
Mark Myatt ${ }^{1}$ * , Tanya Khara ${ }^{2}$, Carmel Dolan ${ }^{2}$, Michel Garenne $3,4,5,6$ and André Briend 7,8 \\ 'Brixton Health, Cilfach Greigiog, Fford Celynin, Llwyngwril, Gwynedd, LL37 2JD, UK: ${ }^{2}$ Emergency Nutrition \\ Network, Oxford, UK: ${ }^{3}$ IRD, UMI Résiliences, Paris, France: ${ }^{4}$ Institut Pasteur, Epidémiologie des Maladies \\ Emergentes, Paris, France: ${ }^{5} \mathrm{FERDI}$, Université d'Auvergne, Clermont-Ferrand, France: ${ }^{6} \mathrm{MRC} /$ Wits Rural Public \\ Health and Health Transitions Research Unit, School of Public Health, Faculty of Health Sciences, University of the \\ Witwatersrand, Johannesburg, South Africa: ${ }^{7}$ School of Medicine, Center for Child Health Research, University of \\ Tampere, Tampere, Finland: ${ }^{8}$ Department of Nutrition, Exercise and Sports, University of Copenhagen, \\ Copenhagen, Denmark
}

Submitted 13 April 2018: Final revision received 28 September 2018: Accepted 16 0ctober 2018: First published online 3 December 2018

\begin{abstract}
Objective: To investigate whether children with concurrent wasting and stunting require therapeutic feeding and to better understand whether multiple diagnostic criteria are needed to identify children with a high risk of death and in need of treatment.

Design: Community-based cohort study, following 5751 children through time. Each child was visited up to four times at 6-month intervals. Anthropometric measurements were taken at each visit. Survival was monitored using a demographic surveillance system operating in the study villages.

Setting: Niakhar, a rural area of the Fatick region of central Senegal.

Participants: Children aged 6-59 months living in thirty villages in the study area. Results: Weight-for-age $Z$-score (WAZ) and mid-upper arm circumference (MUAC) were independently associated with near-term mortality. The lowest WAZ threshold that, in combination with MUAC, detected all deaths associated with severe wasting or concurrent wasting and stunting was WAZ $<-2 \cdot 8$. Performance for detecting deaths was best when only WAZ and MUAC were used. Additional criteria did not improve performance. Risk ratios for near-term death in children identified using WAZ and MUAC suggest that children identified by WAZ $<-2 \cdot 8$ but with $M U A C \geq 115 \mathrm{~mm}$ may require lower-intensity treatment than children identified using MUAC $<115 \mathrm{~mm}$.

Conclusions: A combination of MUAC and WAZ detected all near-term deaths associated with severe anthropometric deficits including concurrent wasting and stunting. Therapeutic feeding programmes may achieve higher impact if WAZ and MUAC admission criteria are used.
\end{abstract}

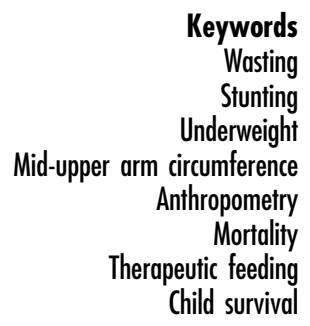

Wasting and stunting are common conditions in children. It is estimated that 52 million children are wasted, 16 million of whom are severely wasted, and 155 million children are stunted $^{(1)}$. Wasting and stunting are implicated in the deaths of almost 2 million children annually and account for over $15 \%$ of all disability-adjusted life years lost in young children ${ }^{(2)}$. These figures are likely to underestimate burden because the number of wasted children present in a population (i.e. prevalent cases) found from unadjusted cross-sectional prevalence estimates will miss many incident cases of an acute condition ${ }^{(3)}$.

Wasting and stunting in children tend to be addressed as separate issues in programmes, policy and research.
Recent reviews have called this separation into question $^{(4-8)}$. Wasting and stunting are often present in the same populations ${ }^{(9)}$ and there is evidence that the two conditions share underlying and immediate causal factors $^{(10,11)}$. Investigation into whether there is a direct causal relationship between wasting and stunting is ongoing and a number of gaps in the evidence base have been identified $^{(12)}$. There has also been recent recognition that children may be both stunted and wasted at the same time ${ }^{(13-15)}$. National estimates of the prevalence of concurrent wasting and stunting in children, calculated for eighty-four countries, range between 0 and $8 \%$, exceeding $5 \%$ in nine countries ${ }^{(16)}$. The factors leading to this state of 
'concurrence' are poorly understood but the available evidence indicates that considerable excess mortality is experienced by these children. The level of excess mortality is not explained by the severity of their wasting and stunting alone. This suggests a multiplicative effect on mortality of having both deficits ${ }^{(17,18)}$. The magnitude of the mortality risk associated with concurrent wasting and stunting is similar to that associated with severe acute malnutrition $(\mathrm{SAM})^{(19)}$. This raises the question of whether these children should be included in therapeutic feeding programmes which historically have treated only children with SAM as currently defined by the WHO and UNICEF using three independent diagnostic criteria:

1. mid-upper arm circumference (MUAC) below $115 \mathrm{~mm}$;

2. weight-for-height $Z$-score (WHZ) below -3.0; and

3. the presence of bilateral nutritional oedema.

The presence of one or more of these criteria is enough to warrant admission into a therapeutic feeding programme ${ }^{(20)}$.

To investigate whether all children with concurrent wasting and stunting (WaSt) require therapeutic feeding and to better understand whether multiple diagnostic criteria are needed to identify children with a high risk of death and in need of treatment, an analysis examining which anthropometric indices are independently associated with near-term mortality is likely to prove a useful approach. The data for this type of analysis should come from a community-based cohort study assessing the risk of death in a representative sample of untreated children. Studies collecting data for such an analysis are no longer possible. The development of communitybased management of acute malnutrition (CMAM) programmes capable of delivering effective SAM treatment at high levels of coverage have made it unethical to leave highrisk children untreated. The aim of the present paper is to describe the results of an analysis examining the ability of different anthropometric case definitions to identify children at high risk of near-term death. The analysis was performed using an existing data set from a historical community-based cohort study that pre-dates the development of CMAM programme models. An earlier analysis of the same data set showed strong relationships between a variety of nutritional indicators and mortality, with the highest populationattributable fractions associated with MUAC and WAZ ${ }^{(21)}$.

\section{Methods}

\section{Data sources}

The analysis presented here is based on a data set collected in 1983 and 1984 in Niakhar, a rural area of the Fatick region of central Senegal. These data have been described in detail elsewhere ${ }^{(22)}$. An open cohort of 5751 children, comprising all children under 5 years of age living in thirty villages in the study area, was followed through time. Each child was visited up to four times at 6-month intervals in May and November of 1983 and 1984.
Anthropometric measurements were taken at each visit. All measurements were taken by the research staff after careful training and standardisation ${ }^{(22,23)}$. Weight was measured to the nearest $10 \mathrm{~g}$ using beam scales (SECA France, Semur en Auxois, France). Length and height were measured to the nearest $1 \mathrm{~mm}$ using 'Harpenden' length/ height measurement boards (Holtain Ltd, Crymych, UK). MUAC was measured to the nearest $1 \mathrm{~mm}$ using nonelastic (fibreglass) tapes. Survival was monitored for all children using a demographic surveillance system operating in the study villages that was established in $1962^{(24)}$. The ages of children at each visit were calculated using the dates of birth taken from this demographic surveillance system and the dates of measurement by research staff. Approval for the original study was obtained from the International Health Department, the Scientific Commission and the Director General of ORSTOM/IRD (the French Institute for Scientific Research Overseas/Research Institute for Development) in France and from the Ministry of Health, Department of Statistics, Census Bureau and ORANA (Office de Recherches sur l'Alimentation et la Nutrition Africaines) in Senegal. Fieldwork was supported by ORSTOM/IRD, ORANA and the European Union's DGXII directorate (grant number TDR-36). Participation in the study was voluntary. Oral informed consent was obtained from principal caregivers. The analysis presented here did not involve new data collection and was done using anonymised records; therefore, it did not require further ethical approval.

Height-for-age, weight-for-age and weight-for-height $Z$-scores (HAZ, WAZ and WHZ, respectively) were calculated using the $\mathrm{WHO}$ growth standards ${ }^{(25)}$. The outcome of interest was death within 183 d (i.e. 6 months) of anthropometric assessment. The analysis was intended to investigate the association between anthropometry and near-term mortality to inform the selection of case-finding and admission criteria for therapeutic feeding programmes. These programmes treat SAM in children aged 6-59 months with the same standard protocol using readyto-use therapeutic foods. These children represent the vast majority of children currently treated in such programmes. Therefore, the current analysis used data only for children aged 6-59 months at the time of anthropometric assessment ( $n$ 5144). The units of analysis used in the analyses are the individual 6-month follow-up episodes (12638 episodes with 304 deaths).

Data management and data analysis were performed using purpose-written $\mathrm{R}$ language (version 3.5.1) scripts managed using the $\mathrm{R}$ Analytic-Flow scientific workflow system (version 3.1.8).

\section{Association between anthropometry and mortality}

Bivariate associations between different anthropometric variables (i.e. HAZ, WAZ, WHZ and MUAC) and death within 6 months of measurement were examined by 
estimating mean values for died and survived follow-up episodes using Student's $t$ test and the common language effect size (probability of superiority) statistic ${ }^{(26)}$, which estimates the probability that a value drawn at random from the survived group will be greater than a value drawn at random from the died group. The null (i.e. no difference) value for the common language effect size statistic is $0 \cdot 5$. Multiple logistic regression was used to distinguish between real and apparent (i.e. due to confounding) associations. All variables with significant associations in the bivariate analyses were entered into the model. Non-significant variables were removed using backwards stepwise elimination with the least significant variable $(P>0.05)$ removed at each step.

\section{Association between anthropometric status and mortality}

Bivariate associations between anthropometric case definitions and death within 6 months of measurement were examined by calculating risk ratios from two-by-two tables. Standard case definitions for severe undernutrition were used;

1. Severely stunted: $H A Z<-3 \cdot 0$.

2. Severely underweight: $\mathrm{WAZ}<-3 \cdot 0$.

3. Severely wasted: $\mathrm{WHZ}<-3 \cdot 0$.

4. Severely low MUAC: MUAC $<115 \mathrm{~mm}$.

An additional anthropometric case definition for being concurrently wasted and stunted (WaSt) was also used;

5. WaSt: $\mathrm{WHZ}<-2 \cdot 0$ and $\mathrm{HAZ}<-2 \cdot 0$.

Multiple logistic regression with death within 6 months of measurement as the outcome, as described above, was used to distinguish between real and apparent (i.e. due to confounding) associations.

\section{Using anthropometry to predict mortality}

The possibility of identifying all, or nearly all, children with severe anthropometric deficits who are likely to die within 6 months of measurement using a combination of anthropometric case definitions was explored using fourdimensional Venn diagrams with sets defined by WAZ, WHZ, MUAC and WaSt case definitions ${ }^{(27)}$. WHZ $<-3 \cdot 0$ and MUAC $<115 \mathrm{~mm}$ case definitions were used because these are commonly used as admission criteria for programmes treating $\mathrm{SAM}^{(20)}$. An appropriate threshold for a WAZ case definition was found by a directed search for the lowest WAZ threshold that, in combination with MUAC, detected all deaths associated with the WHZ and WaSt case definitions. A second analysis used MUAC < $125 \mathrm{~mm}$ instead of MUAC $<115 \mathrm{~mm}$. This threshold is used in programmes integrating treatment of both severe and moderate acute malnutrition ${ }^{(28)}$. Sensitivity, specificity and
Youden's index:

$$
J=\text { Sensitivity }+ \text { Specificity }-1,
$$

were estimated for case definitions based on combinations of WAZ, WHZ, MUAC and WaSt case definitions. Youden's index measures overall performance of a screening or diagnostic test ${ }^{(29)}$. The maximum value of Youden's index occurs for case definitions that optimise differentiating ability when equal weight is given to sensitivity and specificity. Risk ratios for death within 6 months of measurement in the populations selected using different combinations of MUAC and WAZ case definitions identified by the Venn diagram analysis were estimated.

\section{Effects of changing case definitions on programme caseloads}

The effect of changing case definitions on program caseloads was investigated using simple 'what-if?' simulations. The model used was:

$$
\text { Caseload }=\text { Population } \times \text { Prevalence } \times \text { Coverage } \text {. }
$$

The population term was fixed at 17000 assuming a service delivery unit (e.g. a health district) with a total population of 100000 with $17 \%$ of the total population aged between 6 and 59 months. Prevalence and coverage terms were modelled using fuzzy triangular numbers and fuzzy arithmetic with $95 \%$ CI for ratio estimates calculated for a triangular distribution ${ }^{(30,31)}$. Prevalence was modelled using the 25th, 50th and 75th percentiles of prevalence estimates from 2426 nutritional anthropometry surveys from fifty-one countries from 1992 to $2015^{(18)}$. Coverage for programmes admitting using MUAC was modelled using the 25th, 50th and 75th percentiles of coverage estimates from 227 coverage assessments of CMAM programmes from twenty-nine countries from 2009 to 2017 recorded in a database provided by the Coverage Monitoring Network. Recent CMAM programme coverage estimates for cases meeting the $\mathrm{WHZ}<-3$ admission criteria were unavailable. Work on coverage undertaken in pilots of the CMAM programme model found coverage of cases with $\mathrm{WHZ}<-3$ to be consistently low (i.e. $\leq 20 \%$ ). It was assumed that coverage for WHZ cases would be onethird that of coverage for MUAC cases. It was assumed that cases with WAZ $<-2.8$ would be identified by growth monitoring or growth monitoring and promotion (GM/ GMP) programmes. A literature review was undertaken and found useable coverage data for twenty-three GM/ GMP programmes of differing scope and design from fourteen countries ${ }^{(32-39)}$. GM/GMP coverage was defined as a population coverage of more than three measurements in the previous 6 months. It was assumed that coverage in lower socio-economic groups (i.e. those groups most at risk of malnutrition) would be $80 \%$ of reported coverage. It was also assumed that the interface between GM/GMP programmes and CMAM programmes would be imperfect, with $80 \%$ of cases identified by the 
GM/GMP programmes being admitted into CMAM programmes. Coverage of children with WAZ $<-2 \cdot 8$ was modelled using the 25 th, 50 th and 75 th percentiles of the resulting estimates.

\section{Results}

Association between antbropometry and mortality Table 1 shows the results of the analysis of bivariate associations between anthropometric variables and death within 6 months of measurement in the Niakhar cohort data. HAZ, WAZ, WHZ and MUAC were all negatively associated with death within 6 months of measurement. Table 2 shows the results of the multivariate analysis after non-significant variables had been removed. Only WAZ and MUAC were independently associated with death within 6 months of measurement.

\section{Anthropometric status and mortality}

Table 3 shows the results of the analysis of the bivariate associations between anthropometric case definitions and death within 6 months of measurement in the Niakhar cohort data. All anthropometric case definitions were associated with death within 6 months of measurement. Table 4 shows the results of the multivariate analysis after non-significant variables had been removed. Only severe underweight (WAZ <-3.0) and severely low MUAC (MUAC $<115 \mathrm{~mm}$ ) were independently associated with death within 6 months of measurement.

\section{Using antbropometry to predict mortality}

The optimum WAZ threshold (i.e. the lowest WAZ threshold that detected all deaths associated with the WHZ and WaSt case definitions when used in combination with MUAC case definitions) was WAZ $<-2 \cdot 8$. Figure 1 shows the four-dimensional Venn diagram for sets defined using the MUAC $<115 \mathrm{~mm}$, WHZ $<-3 \cdot 0$, WAZ $<-2 \cdot 8$ and WaSt case definitions. The inclusion of $\mathrm{WHZ}<-3.0$ and WaSt did not identify additional deaths when MUAC $<115 \mathrm{~mm}$ and WAZ $<-2 \cdot 8$ were used to select high-risk children. Figure 2 shows the four-dimensional Venn diagram for sets defined using the MUAC $<125 \mathrm{~mm}$, WHZ $<-3 \cdot 0$, WAZ $<-2.8$ and WaSt case definitions. Increasing the MUAC cut-off to $125 \mathrm{~mm}$ identified additional deaths but WAZ $<-2.8$ still identified deaths not identified using MUAC.

Table 5 presents point estimates of sensitivity, specificity and Youden's index for case definitions based on combinations of WAZ, WHZ, MUAC and WaSt case definitions. Overall performance (i.e. simplicity, the number of deaths detected, sensitivity, specificity and Youden's index) was

Table 1 Bivariate associations between anthropometric variables and death within 6 months of measurement among children aged 6-59 months in the Niakhar cohort data, rural Senegal ${ }^{(22)}$

\begin{tabular}{|c|c|c|c|c|c|c|}
\hline Variable & $\begin{array}{l}\text { Outcome after } \\
6 \text { months }\end{array}$ & Mean & $95 \% \mathrm{Cl}$ & $P^{\star}$ & $w \dagger$ & $95 \% \mathrm{Cl}$ \\
\hline $\mathrm{HAZ}$ & $\begin{array}{l}\text { Died } \\
\text { Survived }\end{array}$ & $\begin{array}{l}-1 \cdot 78 \\
-1 \cdot 18\end{array}$ & $\begin{array}{l}-1.92,-1.63 \\
-1.20,-1.16\end{array}$ & $<0.0001$ & 0.63 & $0.62,0.64$ \\
\hline WAZ & $\begin{array}{l}\text { Died } \\
\text { Survived }\end{array}$ & $\begin{array}{l}-2.35 \\
-1.40\end{array}$ & $\begin{array}{l}-2 \cdot 49,-2 \cdot 20 \\
-1.42,-1.38\end{array}$ & $<0.0001$ & 0.71 & $0.70,0.72$ \\
\hline $\mathrm{WHZ}$ & $\begin{array}{l}\text { Died } \\
\text { Survived }\end{array}$ & $\begin{array}{l}-1.56 \\
-0.79\end{array}$ & $\begin{array}{l}-1.72,-1.40 \\
-0.82,-0.77\end{array}$ & $<0.0001$ & 0.66 & $0.65,0.67$ \\
\hline MUAC (mm) & $\begin{array}{l}\text { Died } \\
\text { Survived }\end{array}$ & $\begin{array}{l}132 \cdot 5 \\
143 \cdot 4\end{array}$ & $\begin{array}{l}130 \cdot 8,134 \cdot 1 \\
143 \cdot 1,143 \cdot 6\end{array}$ & $<0.0001$ & 0.70 & $0.69,0.71$ \\
\hline
\end{tabular}

HAZ, height-for-age Z-score; WAZ, weight-for-age Z-score; WHZ, weight-for-height Z-score; MUAC, mid-upper arm circumference.

${ }^{*} P$ value for Student's $t$ test.

†Common language effect size (probability of superiority) statistic. The statistic estimates the probability that a value drawn at random from the survived group will be greater than a value drawn at random from the died group. The null (i.e. no difference) value is 0.5 . The statistic is based on comparison of $c .123$ million pairs of values.

Table 2 Independent associations between anthropometric variables and death within 6 months of measurement* among children aged 6-59 months in the Niakhar cohort data, rural Senegal ${ }^{(22)}$

\begin{tabular}{lccc}
\hline Variable & OR & $95 \% \mathrm{Cl}$ & $P$ \\
\hline WAZ & 0.75 & $0.64,0.87$ & $<0.0001$ \\
MUAC (mm) $\dagger$ & 0.97 & $0.95,0.98$ & $<0.0001$ \\
\hline
\end{tabular}

WAZ, weight-for-age Z-score; MUAC, mid-upper arm circumference.

*Variables remaining in the model after non-significant variables were removed using backwards stepwise elimination. TWAZ is recorded in Z-scores but MUAC is recorded in millimetres. A comparable OR for MUAC and death within 6 months of measurement is approximately $0.97^{\operatorname{SD}(\text { MUAC) }} \approx 0.97^{13.72} \approx 0.66$. 
Table 3 Anthropometric case status and relative risk of death within 6 months of measurement (bivariate analysis) among children aged 6-59 months in the Niakhar cohort data, rural Senegal ${ }^{(22)}$

\begin{tabular}{lccccc}
\hline Anthropometric status & Class & Case definition & $\mathrm{RR}$ & $95 \% \mathrm{Cl}$ & $P^{*}$ \\
\hline Stunted & Severe & HAZ $<-3.0$ & 2.79 & $2.10,3.69$ & $<0.0001$ \\
Underweight & Severe & WAZ $<-3.0$ & 3.97 & $3.13,5.02$ & $<0.0001$ \\
Wasted by WHZ & Severe & WHZ $<-3.0$ & 4.47 & $3.26,6.12$ & $<0.0001$ \\
Low MUAC & Severe & MUAC $<115 \mathrm{~mm} \dagger$ & 5.09 & $3.58,7.23$ & $<0.0001$ \\
WaSt & All & WHZ $<-2.0 \&$ HAZ $<-2.0$ & 4.08 & $3.13,5.33$ & $<0.0001$ \\
\hline
\end{tabular}

$\mathrm{RR}$, risk ratio; WHZ, weight-for-height $Z$-score; MUAC, mid-upper arm circumference; WaSt, concurrent wasting and stunting; HAZ, height-for-age Z-score; WAZ, weight-for-age Z-score; CMAM, community management of acute malnutrition.

${ }^{*} P$ value for Fisher's exact test.

†MUAC $<115 \mathrm{~mm}$ is commonly used for case finding in the community for admission to CMAM programmes. It is the primary CMAM programme admission criterion in many countries.

Table 4 Independent associations between anthropometric case status and death within 6 months of measurement* among children aged 6-59 months in the Niakhar cohort data, rural Senegal ${ }^{(22)}$

\begin{tabular}{lccccc}
\hline Anthropometric status & Class & Case definition & OR & $95 \% \mathrm{Cl}$ & $P$ \\
\hline Underweight & Severe & WAZ $<-3.0$ & 3.51 & $2.65,4.65$ & $<0.0001$ \\
Low MUAC & Severe & MUAC $<115 \mathrm{~mm} \dagger$ & 2.20 & $1.41,3.42$ & $<0.0005$
\end{tabular}

MUAC, mid-upper arm circumference; WAZ, weight-for-age Z-score; CMAM, community management of acute malnutrition.

*Variables remaining in the model after non-significant variables were removed using backwards stepwise elimination. †MUAC $<115 \mathrm{~mm}$ is commonly used for case finding in the community for admission to CMAM programmes. It is the primary CMAM programme admission criterion in many countries.

better for case definitions based on WAZ and MUAC. Adding WaSt or WHZ criteria resulted in slightly degraded performance (i.e. slightly reduced specificity).

Risk ratios for death within 6 months of measurement in the populations selected using combinations of MUAC and WAZ case definitions identified by the Venn diagram analysis - i.e. MUAC $<115 \mathrm{~mm} ; \quad$ MUAC $<125 \mathrm{~mm}$ and MUAC $\geq 115 \mathrm{~mm} ; \quad$ WAZ $<-2.8$ and $M U A C \geq 115 \mathrm{~mm}$; WAZ $<-2 \cdot 8$ and MUAC $\geq 125 \mathrm{~mm}$ - are presented in Fig. 3.

\section{Effects of changing case definitions on programme caseloads}

Table 6 presents the results of the simple 'what-if?' simulations of the effect of changing case definitions on programme caseload. The simulated caseload for programmes admitting using MUAC $<115 \mathrm{~mm}$ and WHZ $<-3$ was 123 (95\% CI 68, 260) cases per 17000 population. The simulated caseload for programmes admitting using MUAC $<115 \mathrm{~mm}$ or WAZ $<-2 \cdot 8$ was 355 (95\% CI 179, 644) cases per 17000 population. The simulated caseload for programmes using MUAC $<115$ mm or WAZ $<-2 \cdot 8$ was $2 \cdot 89$ (95\% CI $1.38,13.41)$ times larger than the simulated caseload for programmes admitting using MUAC $<115 \mathrm{~mm}$ or $\mathrm{WHZ}<-3$. The simulated caseload for programmes admitting using MUAC $<115 \mathrm{~mm}$ only was 92 (95\% CI 50, 169) cases per 17000 population. The simulated caseload for programmes admitting using MUAC $<125 \mathrm{~mm}$ only was 488 (95\% CI 300, 885) per 17000 population. The simulated caseload for programmes using MUAC $<125 \mathrm{~mm}$ only was
5.30 (95\% CI 2.79, 25.54) times larger than the simulated caseload for programmes admitting using MUAC $<115 \mathrm{~mm}$ only.

\section{Discussion}

The analysis presented here found that MUAC and WAZ were independently associated with death within 6 months of measurement. A previous multivariate (i.e. logistic regression) analysis of community-based cohort data from Bangladesh also reported that WHZ did not help identify high-risk children when MUAC was already in the model ${ }^{(40)}$.

A combination of MUAC and WAZ was able to detect all near-term deaths associated with severe anthropometric deficits including children who were simultaneously wasted and stunted (WaSt). This suggests that the MUAC admission criterion in therapeutic feeding programmes should be retained and that consideration should be given to replacing the current WHZ admission criterion with a WAZ admission criterion.

WHZ $<-3.0$ was part of the clinical definition of severe malnutrition used in the 1999 WHO manual for the management of severe malnutrition ${ }^{(41)}$. This was based on the understanding that it reflected recent severe weight loss ${ }^{(41)}$. MUAC was introduced a decade later because it is easy to use in the community and is better at identifying children with a high risk of death than $\mathrm{WHZ}^{(20,42)}$. Using WHZ requires measurement of length or height. This is time-consuming, requires the presence of at least two health workers and requires length/height boards which 


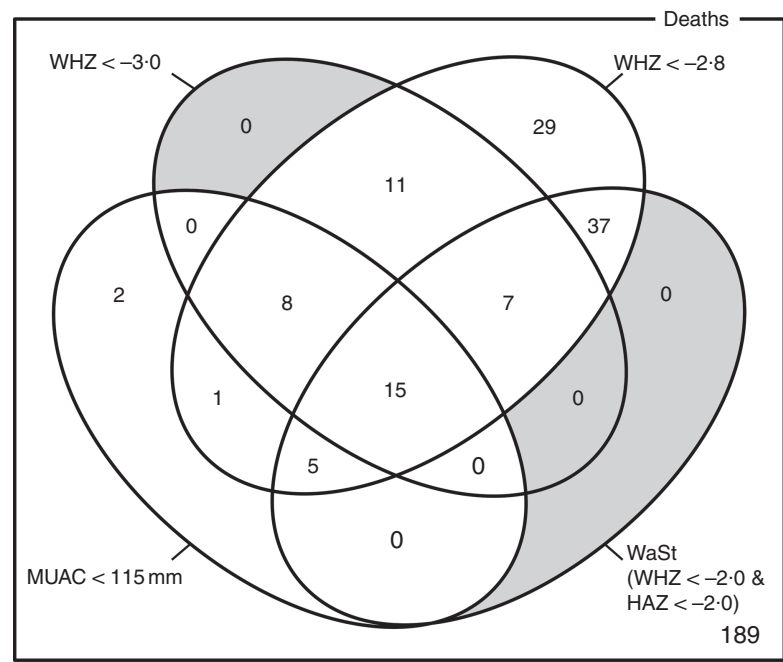

Fig. 1 Numbers of deaths identified using MUAC $<115 \mathrm{~mm}$, $W H Z<-3.0, W A Z<-2.8$ and WaSt case definitions among children aged 6-59 months in the Niakhar cohort data, rural Senegal ${ }^{(22)}$. The shaded area shows cells outside the union of the MUAC $<115 \mathrm{~mm}$ and $\mathrm{WAZ}<-2.8$ sets. MUAC $<115 \mathrm{~mm}$ or $W A Z<-2.8$ detected all deaths associated with WaSt and with $\mathrm{WHZ}<-3 \cdot 0$. MUAC $<115 \mathrm{~mm}$ or WAZ $<-2.8$ detected more deaths than MUAC $<115 \mathrm{~mm}$ or $\mathrm{WHZ}<-3.0$ (MUAC, mid-upper arm circumference; WHZ, weight-for-height Z-score; WAZ, weight-for-age Z-score; WaSt, concurrent wasting and stunting; $\mathrm{HAZ}$, height-for-age Z-score)

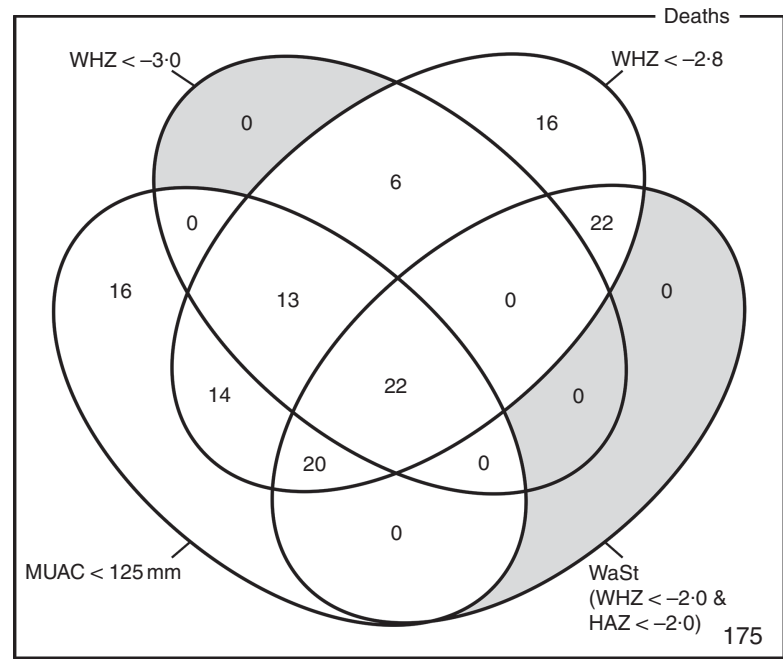

Fig. 2 Numbers of deaths identified using $M U A C<125 \mathrm{~mm}$, $W H Z<-3.0, W A Z<-2.8$ and WaSt case definitions among children aged 6-59 months in the Niakhar cohort data, rural Senegal ${ }^{(22)}$. The shaded area shows cells outside the union of the MUAC $<125 \mathrm{~mm}$ and $W A Z<-2.8$ sets. MUAC $<125 \mathrm{~mm}$ or WAZ $<-2.8$ detected all deaths associated with WaSt and with $\mathrm{WHZ}<-3 \cdot 0$. MUAC $<125 \mathrm{~mm}$ or $\mathrm{WAZ}<-2.8$ detected more deaths than MUAC $<115 \mathrm{~mm}$ or $\mathrm{WHZ}<-3.0$ (MUAC, mid-upper arm circumference; $W H Z$, weight-for-height $Z$-score; WAZ, weight-for-age Z-score; WaSt, concurrent wasting and stunting; HAZ, height-for-age Z-score)

are not available in many health facilities. Increasing the MUAC cut-off to $120 \mathrm{~mm}$ is likely to be more effective for identifying high-risk children than using the current combination of MUAC and $\mathrm{WHZ}^{(43)}$. If two anthropometric criteria are to be used to define severe undernutrition, it is not clear that WHZ in addition to MUAC is a good choice. Community-based cohort studies have repeatedly shown that WHZ is the least useful anthropometric criterion for identifying high-risk children and that WAZ performs significantly and consistently better at this task ${ }^{(42-45)}$. Recent analyses show that low WHZ is associated with a high risk of death only when it is also associated with stunting defined by low HAZ ${ }^{(17,18)}$. This is concurrent wasting and stunting (WaSt) ${ }^{(18)}$. Recent work on the descriptive epidemiology of multiple anthropometric deficits using data from almost 1.8 million children living in fifty-one countries reported that all children who were concurrently wasted and stunted were also underweight, the maximum possible WAZ for children with WaSt was below $-2 \cdot 35$ when the WHO growth standards are used, and cases of WaSt can be detected with excellent sensitivity and specificity using $\mathrm{WAZ}^{(18)}$. This, together with the difficulty of providing good spatial and temporal coverage of the height measurements required for calculating HAZ and WHZ, means that WAZ may be used as a practicable indicator of WaSt. WAZ is easier to measure than WHZ because it does not require height or length to be measured. It does, however, lack the simplicity and low cost of MUAC. Any anthropometric indicator that includes an age component, such as WAZ, requires that age be ascertained accurately $^{(46)}$. This is unlikely to be problem in GM/GMP programmes. Lack of simplicity, relatively high cost and the need to ascertain age accurately are likely to impair the utility of WAZ for detecting cases in the community ${ }^{(42)}$. Depending on the thresholds used, MUAC has higher attributable risks than $\mathrm{WAZ}^{(21)}$. This means that WAZ may be of most value when it is already being measured in (e.g.) GM/GMP programmes. Linkages between GM/GMP programmes and therapeutic feeding programmes could be forged and children with MUAC $<115 \mathrm{~mm}$ or WAZ $<$ $-2 \cdot 8$ referred to therapeutic feeding programmes from GM/GMP programmes (see Fig. 4).

Increases in caseload may not translate into similar increases in workload or costs if the additional cases identified by WAZ require lower-intensity treatment (e.g. smaller quantities of therapeutic feeding products and reduced frequency of contact) than cases identified by MUAC. A similar approach is used in programmes integrating treatment of both severe and moderate acute malnutrition ${ }^{(28)}$. Examination of mortality risks (Fig. 3) suggests that a low-intensity treatment may be appropriate for cases identified using WAZ. Simulation of caseloads for various scenarios (Table 6) suggests that the increase in caseload associated with replacing WHZ with WAZ is unlikely to be larger than that associated with moving from MUAC $<115 \mathrm{~mm}$ to MUAC $<125 \mathrm{~mm}$ which has proved to be manageable ${ }^{(28)}$.

Current programme designs tend to focus on either acute malnutrition (wasting) or chronic malnutrition 
Table 5 Point estimates of sensitivity, specificity and Youden's index for detecting near-term deaths of different screening/admission criteria based on combinations of WAZ, WHZ, MUAC and WaSt case definitions among children aged 6-59 months in the Niakhar cohort data, rural Senegal ${ }^{(22)}$

\begin{tabular}{|c|c|c|c|c|}
\hline Screening/admission criteria* & Deaths detected & Sensitivity & Specificity & Youden's index \\
\hline WaSt† & 64 & 0.2105 & 0.9424 & 0.1529 \\
\hline$W A Z<-2.8$ & 113 & 0.3717 & 0.8704 & 0.2421 \\
\hline WaSt or WAZ $<-2 \cdot 8$ & 113 & 0.3717 & 0.8675 & 0.2392 \\
\hline $\mathrm{WHZ}<-3.0$ & 41 & 0.1349 & 0.9688 & 0.1037 \\
\hline WaSt or $\mathrm{WHZ}<-3.0$ & 83 & 0.2730 & 0.9260 & 0.1990 \\
\hline$W A Z<-2.8$ or $W H Z<-3.0$ & 113 & 0.3717 & 0.8670 & 0.2387 \\
\hline WaSt or WAZ $<-2.8$ or $W H Z<-3.0$ & 113 & 0.3717 & 0.8640 & 0.2357 \\
\hline MUAC $<115 \mathrm{~mm}$ & 31 & 0.1020 & 0.9801 & 0.0821 \\
\hline WaSt or MUAC $<115 \mathrm{~mm}$ & 75 & 0.2467 & 0.9353 & 0.1820 \\
\hline WAZ $<-2.8$ or MUAC $<115 \mathrm{~mm} \ddagger$ & 115 & 0.3783 & 0.8677 & 0.2460 \\
\hline WaSt or WAZ $<-2.8$ or MUAC $<115 \mathrm{~mm}$ & 115 & 0.3783 & 0.8648 & 0.2431 \\
\hline $\mathrm{WHZ}<-3.0$ or $\mathrm{MUAC}<115 \mathrm{~mm}$ & 49 & 0.1612 & 0.9604 & 0.1215 \\
\hline WaSt or $\mathrm{WHZ}<-3.0$ or MUAC $<115 \mathrm{~mm}$ & 86 & 0.2829 & 0.9223 & 0.2052 \\
\hline $\mathrm{WAZ}<-2.8$ or $\mathrm{WHZ}<-3.0$ or $\mathrm{MUAC}<115 \mathrm{~mm}$ & 115 & 0.3783 & 0.8646 & 0.2429 \\
\hline WaSt or WAZ $<-2.8$ or $\mathrm{WHZ}<-3.0$ or $\mathrm{MUAC}<115 \mathrm{~mm}$ & 115 & 0.3783 & 0.8617 & 0.2400 \\
\hline MUAC $<125 \mathrm{~mm}$ & 85 & 0.2796 & 0.9180 & 0.1976 \\
\hline WaSt or MUAC $<125 \mathrm{~mm}$ & 107 & 0.3520 & 0.8932 & 0.2452 \\
\hline WAZ $<-2.8$ or $\mathrm{MUAC}<125 \mathrm{~mm}$ & 129 & 0.4243 & 0.8455 & 0.2698 \\
\hline WaSt or WAZ $<-2.8$ or MUAC $<125 \mathrm{~mm}$ & 129 & 0.4243 & 0.8427 & 0.2671 \\
\hline $\mathrm{WHZ}<-3.0$ or $\mathrm{MUAC}<125 \mathrm{~mm}$ & 91 & 0.2993 & 0.9085 & 0.2079 \\
\hline WaSt or $\mathrm{WHZ}<-3.0$ or MUAC $<125 \mathrm{~mm}$ & 113 & 0.3717 & 0.8858 & 0.2576 \\
\hline $\mathrm{WAZ}<-2.8$ or $\mathrm{WHZ}<-3.0$ or $\mathrm{MUAC}<125 \mathrm{~mm}$ & 129 & 0.4243 & 0.8430 & 0.2674 \\
\hline WaSt or WAZ $<-2.8$ or $W H Z<-3.0$ or $M U A C<125 \mathrm{~mm}$ & 129 & 0.4243 & 0.8403 & 0.2646 \\
\hline
\end{tabular}

WAZ, weight-for-age Z-score; WHZ, weight-for-height Z-score; MUAC, mid-upper arm circumference; WaSt, concurrent wasting and stunting.

${ }^{*}$ The screening criteria (case definition) used to detect children with high risk of death.

tWaSt refers to $\mathrm{WHZ}<-2.0$ and $\mathrm{HAZ}<-2.0$.

$\ddagger$ The best-performing (i.e. in terms of the number of indicators in the screening/admission criteria (fewer is better), the number of deaths detected (more is better) and Youden's index (higher is better) screening criteria found in this analysis.

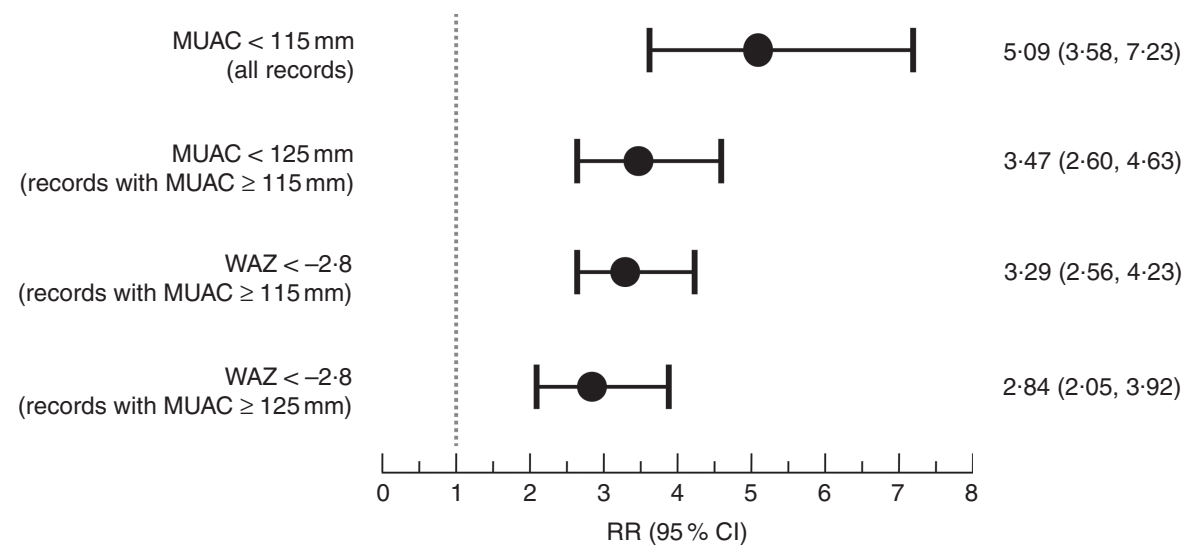

Fig. 3 Risk ratios (RR) for death within 6 months of measurement (with their $95 \% \mathrm{Cl}$ represented by horizontal bars) associated with different MUAC and/or WAZ case definitions among children aged 6-59 months in the Niakhar cohort data, rural Senegal ${ }^{(22)}$ (MUAC, mid-upper arm circumference; WAZ, weight-for-age Z-score

(stunting). The distinction between acute and chronic malnutrition appears arbitrary ${ }^{(4-11)}$. An extensive literature review found no risk factor for wasting that was not also associated with stunting ${ }^{(10)}$. Being wasted is a risk factor for being stunted and vice versa ${ }^{(18)}$. The analysis presented here avoids the distinction between acute and chronic malnutrition and, following earlier work on admission criteria for CMAM programmes, focuses on near-term mortality risk associated with one or more anthropometric deficits ${ }^{(42)}$. Programmes admitting using MUAC and WAZ, both of which are associated with wasting and stunting, would treat children with anthropometric deficits associated with near-term mortality rather than just 'acute malnutrition'.

Further work is required before the findings of the work reported here can be applied. This includes:

1. Repeating the current analysis using data from community-based cohort studies of untreated children 
Table 6 Results of the simple 'what-if?' simulations of the effect of changing case definitions on programme caseloads

\begin{tabular}{lcccc}
\hline Admission criteria & Population & Prevalence $^{*}(\%)$ & Coverage $^{*}(\%)$ & Caseload $^{*}$ \\
\hline MUAC $<115 \mathrm{~mm}$ & 17000 & $(0 \cdot 6,1 \cdot 3,2 \cdot 5)$ & $(32 \cdot 4,41 \cdot 6,52 \cdot 0)$ & $(33,92,221)$ \\
MUAC $<125 \mathrm{~mm}$ & & $(4 \cdot 2,6 \cdot 9,11 \cdot 1)$ & & $(231,488,981)$ \\
WHZ $<-3 \&$ MUAC $\geq 115 \mathrm{~mm} \dagger$ & & $(0 \cdot 7,1 \cdot 3,2 \cdot 4)$ & $(10.8,13 \cdot 9,17 \cdot 3)$ & $(13,31,71)$ \\
WAZ $<-2.8 \&$ MUAC $\geq 115 \mathrm{~mm} \dagger$ & & $(4 \cdot 1,7.1,10 \cdot 9)$ & $(12 \cdot 5,21 \cdot 8,26 \cdot 8)$ & $(87,263,497)$ \\
\hline
\end{tabular}

*Parameter values and results are presented as fuzzy triangular numbers.

†Cases additional to those found using the MUAC $<115 \mathrm{~mm}$ case definition

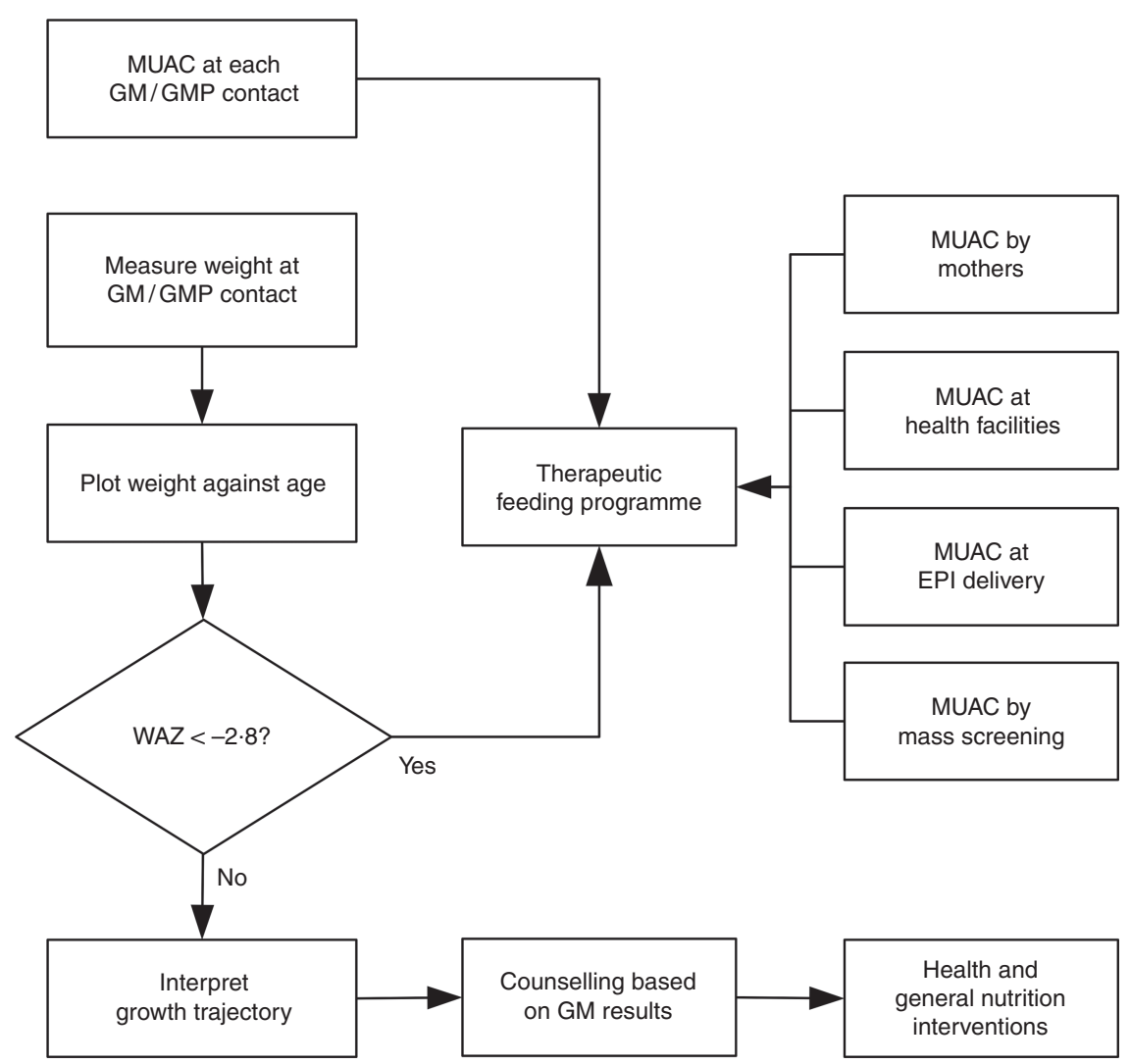

Fig. 4 Delivery model of a programme linking GM/GMP and therapeutic feeding programmes enabling the use of MUAC and WAZ admission criteria in therapeutic feeding programmes (GM, growth monitoring; GMP, growth monitoring and promotion; MUAC, midupper arm circumference; WAZ, weight-for-age Z-score; EPI, expanded programme of immunisation)

from different contexts. This could further inform the selection of a usefully sensitive and specific (i.e. with regard to mortality) case defining WAZ threshold.

2. Ascertaining the intensity and duration of treatment required to treat children identified using WAZ as well as appropriate discharge criteria.

3. Operational research on linking GM/GMP and therapeutic feeding programmes.

The last two issues may be best addressed by small-scale (i.e. single health district) field studies. Such studies would also allow additional key programme planning data such as cost-effectiveness (e.g. cost per cure, cost per life saved, cost per disability-adjusted life year averted) and caseloads to be collected.

\section{Limitations}

The analysis reported here uses data from a single study in a single location. Repeating the current analysis using data from community-based cohort studies of untreated children from different contexts would provide more compelling evidence. Nine additional cohort studies of similar design to the Niakhar study from Bangladesh, Ghana, Guinea Bissau, India, Indonesia, Nepal, Peru, Philippines and Sudan with archived data have been identified and efforts are underway to secure access to these data $^{(17,19,47-53)}$. The caseload simulations are crude, and results should be treated as broadly indicative of the magnitude and direction of the effect of changing case definitions on programme caseloads. 


\section{Conclusions}

Therapeutic feeding programmes concerned with lowering the risk of near-term mortality may achieve higher impact if a combination of MUAC and WAZ admission criteria are used. More work is required before this can be considered as a general recommendation.

\section{Acknowledgements}

Acknowledgements: The authors thank Benard Maire and Olivier Fontaine for their contributions during the initial data collection and earlier analyses of the Niakhar cohort data. Financial support: The original Niakhar study was funded by the European Union, DG-XII (grant number TDR-36). The work reported here was funded by the US Agency for International Development (USAID) (grant number ENN AID-OFDA-G-15-00190) and Irish Aid. The contents of this paper are the responsibility of the authors and do not necessarily reflect the views of USAID or the US Government, nor those of Irish Aid or the Irish Government. The funders had no role in study design; collection, analysis and interpretation of data; the decision to publish; or preparation of the manuscript. Conflict of interest: The authors declare that they have no conflicts of interest. Authorship: The study was conceived and designed by A.B., C.D., M.G., M.M. and T.K. M.G. was the principal investigator of the original Niakhar study and supported the work by contributing data, advising on the analysis of the data and drafting of this article. The data were analysed by M.M. with input from A.B., M.G. and T.K. M.M. and T.K. wrote the first draft of the manuscript and are responsible for the final content. All authors contributed to the article and read and approved the final manuscript. Ethics of buman subject participation: The Niakhar study was conducted with the approval of the Ministry of Health of Senegal.

\section{References}

1. UNICEF, World Health Organization \& The World Bank (2017) Levels and Trends in Child Malnutrition. UNICEF/ WHO/World Bank Group Joint Child Malnutrition Estimates. Key Findings of the 2017 Edition. New York/Geneva/Washington, DC: UNICEF/WHO/World Bank.

2. Black RE, Victora CG, Walker SP et al. (2013) Maternal and child undernutrition and overweight in low-income and middle-income countries. Lancet 382, 427-451.

3. Hure A, Oldmeadow O \& Attia J (2016) Invited commentary: improving estimates of severe acute malnutrition requires more data. Am J Epidemiol 184, 870-872

4. Shoham J, Dolan C \& Gostelow (2013) The Management of Acute Malnutrition at Scale: A Review of Donor and Government Financing Arrangements. Summary Report. Oxford: Emergency Nutrition Network.

5. Menon P \& Stoltzfus RJ (2012) Building convergence in science, programs, and policy actions on child undernutrition: symposium rationale and overview. Adv Nutr $\mathbf{3}$, 224-226.
6. Bergeron G \& Castleman T (2012) Program responses to acute and chronic malnutrition: divergences and convergences. Adv Nutr 3, 242-249.

7. Khara T \& Dolan C (2014) The Relationship Between Wasting and Stunting, Policy, Programming and Research Implications. Technical Briefing Paper. Oxford: Emergency Nutrition Network.

8. Briend A, Khara TN \& Dolan C (2015). Wasting and stunting - similarities and differences; policy and programmatic implications. Food Nutr Bull 36, 1 Suppl., S15-S23.

9. Victora CG (1992) The association between wasting and stunting: an international perspective. $J$ Nutr $\mathbf{1 2 2}$, 1105-1110.

10. Martorell R \& Young MF (2012) Patterns of stunting and wasting: potential explanatory factors. Adv Nutr $\mathbf{3}$, 227-233.

11. Christian P, Lee SE, Donahue Angel M et al. (2013) Risk of childhood undernutrition related to small-for-gestational age and preterm birth in low- and middle-income countries. Int J Epidemiol 42, 1340-1355.

12. Angood C, Khara T, Dolan C et al.; WaSt Technical Interest Group (2016) Research priorities on the relationship between wasting and stunting. PLOS One 11, e0153221.

13. UNICEF, World Health Organization \& The World Bank (2016) Levels and Trends in Child Malnutrition. WHO/ UNICEF/World Bank Group Joint Child Malnutrition Estimates. Key Findings of the 2016 Edition. New York/Geneva/Washington, DC: UNICEF/WHO/World Bank.

14. International Food Policy Research Institute (2015) Global Nutrition Report 2015: Actions and Accountability to Advance Nutrition and Sustainable Development. Washington, DC: IFPRI.

15. International Food Policy Research Institute (2016) Global Nutrition Report 2016: From Promise to Impact: Ending Malnutrition by 2030. Washington, DC: IFPRI.

16. Khara T, Mwangome M, Ngari M et al. (2018) Children concurrently wasted and stunted: a meta-analysis of prevalence data of children 6-59 months from 84 countries. Matern Child Nutr 14, e12516.

17. McDonald CM, Olofin I, Flaxman S et al. (2013) The effect of multiple anthropometric deficits on child mortality: metaanalysis of individual data in 10 prospective studies from developing countries. Am J Clin Nutr 97, 896-901.

18. Myatt M, Khara T, Schoenbuchner S et al. (2018) Children who are both wasted and stunted are also underweight and have a high risk of death: a descriptive epidemiology of multiple anthropometric deficits using data from 51 countries. Arch Public Health 76, 28.

19. Olofin I, McDonald CM, Ezzati M et al. (2013) Associations of suboptimal growth with all-cause and cause-specific mortality in children under five years: a pooled analysis of ten prospective studies. PLoS One 8, e64636.

20. World Health Organization \& UNICEF (2009) WHO Child Growth Standards and the Identification of Severe Acute Malnutrition: A Joint Statement by the World Health Organisation and the United Nations Children's Fund. Geneva: WHO.

21. Garenne M, Maire B, Fontaine O et al. (2006) Distributions of mortality risk attributable to low nutritional status in Niakhar, Senegal. J Nutr 136, 2893-2900.

22. Garenne M, Maire B, Fontaine O et al. (1987) Risques de décès associés à différents états nutritionnels chez l'enfant d'age préscolaire. Dakar/Paris: ORSTOM/ORANA.

23. Briend A, Garenne M, Maire B et al. (1989) Nutritional status, age and survival: the muscle mass hypothesis. Eur J Clin Nutr 43, 715-726.

24. Cantrelle P (1969) Etude démographique dans la région du Siné Saloum (Sénégal); état civil et observations démographiques. Travaux et documents de l'ORSTOM no. 1. Paris: ORSTOM. 
25. World Health Organization (2007) WHO Child Growth Standards: Length-for-Height, Length-for-Age, Weight-forAge, Weight-for-Length, Weight-for-Height and Body Mass Index-for-Age: Methods and Development. Geneva: WHO.

26. McGraw KO \& Wong SP (1992) A common language effect size statistic. Psychol Bull 111, 361-365.

27. Venn J (1880) On the diagrammatic and mechanical representation of propositions and reasonings. Lond Edinb Dubl Phil Mag J Sci 10, 1-18.

28. Maust A, Koroma AS, Abla C et al. (2015) Severe and moderate acute malnutrition can be successfully managed with an integrated protocol in Sierra Leone. J Nutr 145, 2604-2609.

29. Youden WJ (1950) Index for rating diagnostic tests. Cancer 3, 32-35.

30. Kaufman A \& Gupta MM (1985) Introduction to Fuzzy Arithmetic: Theory and Applications. New York: Van Nostrum Rheinhold.

31. Forbes C, Evans M, Hastings N et al. (2011) Statistical Distributions, 4th ed. Hoboken, NJ: John Wiley and Sons.

32. Pearson R (1995) Thematic Evaluation of UNICEF Support to Growth Monitoring. Evaluation and Research Working Paper Series no. 2. New York: UNICEF.

33. Schoeman SE, Dhansay MA, Fincham JE et al. (2003) A community-based growth monitoring model to complement facility-based nutrition and health practices in a semi-urban community in South Africa. S Afr J Clin Nutr 16, 126-132.

34. Sahanggamu PD, Purnomosari L \& Dillon D (2017) Information exposure and growth monitoring favour child nutrition in rural Indonesia. Asia Pac J Clin Nutr 26, 313-316.

35. Faber M, Phungula MAS, Kvalsvig JDA et al. (2003) Acceptability of community-based growth monitoring in a rural village in South Africa. Food Nutr Bull 24, 350-359.

36. World Health Organization (2013) Essential Nutrition Actions: Improving Maternal, Newborn, Infant and Young Child Health and Nutrition. Geneva: WHO.

37. Ettyang GA (1988) The Evaluation of a Nutritional Surveillance, Growth Monitoring and Promotion Programme: An Overview of the Coverage and Quality of Services Provided in Vibiga Division of Kakamega District, Kenya. Nairobi: University of Nairobi.

38. Karim F, Huq N, Brown L et al. (1994) Growth Monitoring in the Context of a Primary Health Care Programme. Tokyo: United Nations University.

39. Ngirabega JDD, Leonard $\mathrm{W}$, Munyanshongore $\mathrm{C}$ et al. (2010) Utilization of community based growth monitoring services by eligible children in rural Rwanda. Rwanda MedJ 68, 40-47.

40. Alam N, Wojtyniak B \& Rahaman MM (1989) Anthropometric indicators and risk of death. Am J Clin Nutr 49, 884-888.
41. World Health Organization (1999) Management of Severe Malnutrition: A Manual for Physicians and Other Senior Health Workers. Geneva: WHO.

42. Myatt M, Khara T \& Collins S (2006) A review of methods to detect cases of severely malnourished children in the community for their admission into community-based therapeutic care programs. Food Nutr Bull 27, 3 Suppl., S7-S23.

43. Briend A, Maire B, Fontaine O et al. (2012) Mid-upper arm circumference and weight-for-height to identify high-risk malnourished under-five children. Matern Child Nutr 8, 130-133.

44. Pelletier DL (1994) The relationship between child anthropometry and mortality in developing countries: implications for policy, programs and future research. $J$ Nutr 124, 10 Suppl., 2047S-2081S

45. Briend A, Mwangome MK \& Berkley JA (2017) Using midupper arm circumference to detect high-risk malnourished patients in need of treatment. In Handbook of Famine, Starvation, and Nutrient Deprivation: From Biology to Policy, pp. 1-17 [VR Preedy and VB Patel, editors]. Cham: Springer International Publishing.

46. Bairagi R (1986) Effects of bias and random error in anthropometry and in age on estimation of malnutrition. $\mathrm{Am}$ J Epidemiol 123, 185-191.

47. Arifeen S, Black RE, Antelman G et al. (2001) Exclusive breastfeeding reduces acute respiratory infection and diarrhea deaths among infants in Dhaka slums. Pediatrics $\mathbf{1 0 8}$, E67.

48. Anon. (1998) Randomised trial to assess benefits and safety of vitamin A supplementation linked to immunisation in early infancy. WHO/CHD Immunisation-Linked Vitamin A Supplementation Study Group. Lancet 352, 1257-1263.

49. Mølbak K, Aaby P, Ingholt L et al. (1992) Persistent and acute diarrhoea as the leading causes of child mortality in urban Guinea Bissau. Trans $R$ Soc Trop Med Hyg 86, 216-220.

50. Katz J, West KP Jr, Tarwotjo I et al. (1989) The importance of age in evaluating anthropometric indexes for predicting mortality. Am J Epidemiol 130, 1219-1226.

51. West KP Jr, Pokhrel RP, Katz J et al. (1991) Efficacy of vitamin A in reducing preschool child mortality in Nepal. Lancet 338, 67-71.

52. Adair L, Popkin BM, VanDerslice J et al. (1993) Growth dynamics during the first two years of life: a prospective study in the Philippines. Eur J Clin Nutr 47, 42-51.

53. Fawzi WW, Herrera MG, Spiegelman DL et al. (1997) A prospective study of malnutrition in relation to child mortality in the Sudan. Am J Clin Nutr 65, 1062-1069. 